University of Nebraska - Lincoln

DigitalCommons@University of Nebraska - Lincoln

6-6-1975

\title{
Path Integrals with Arbitrary Generators and the Eigenfunction Problem
}

\author{
William B. Campbell \\ University of Nebraska-Lincoln, wcambell2@unl.edu \\ Paul Finkler \\ University of Nebraska-Lincoln, pfinkler1@unl.edu \\ C. E. Jones \\ University of Nebraska-Lincoln \\ M. N. Misheloff \\ University of Nebraska-Lincoln
}

Follow this and additional works at: https://digitalcommons.unl.edu/physicsfinkler

Part of the Physics Commons

Campbell, William B.; Finkler, Paul; Jones, C. E.; and Misheloff, M. N., "Path Integrals with Arbitrary Generators and the Eigenfunction Problem" (1975). Paul Finkler Papers. 9.

https://digitalcommons.unl.edu/physicsfinkler/9

This Article is brought to you for free and open access by the Research Papers in Physics and Astronomy at DigitalCommons@University of Nebraska - Lincoln. It has been accepted for inclusion in Paul Finkler Papers by an authorized administrator of DigitalCommons@University of Nebraska - Lincoln. 


\title{
Path Integrals with Arbitrary Generators and the Eigenfunction Problem
}

\author{
W. B. Campbell, P. Finkler, C. E. Jones, ${ }^{*}$ and M. N. Misheloff* \\ Behlen Laboratory of Physics, University of Nebraska, Lincoln, Nebraska 68508
}

Received June 6, 1975

\begin{abstract}
We generalize the path integral formalism of quantum mechanics to include the use of arbitrary infinitesimal generators, thus providing explicit expressions for solutions of a wide class of differential equations. In particular, we develop a method of calculating the eigenfunctions of a large class of operators.
\end{abstract}

\section{INTRODUCTION}

As they are usually applied, path integral techniques are used to study the time development of both nonrelativistic and relativistic quantum-mechanical systems [1-3]. Several years ago, Gutzwiller [4] developed a technique to obtain the energy eigenvalues and eigenfunctions of bound states from the original path integral expressions for Green's functions. Path integral techniques also can be immediately generalized to provide explicit expressions for the matrix elements of an arbitrary infinitesimally generated unitary transformation.

In this paper we show that path integral techniques can be used to study the effects of a much larger class of transformations. Our discussion is expressed in the language of differential operators. We do not restrict our considerations to transformations on a Hilbert space. Many of our results, therefore, cannot be given a quantum-mechanical interpretation; however, any of our results which can be given a meaning in a Hilbert space is quantum-mechanically correct. We use our generalization of path integral techniques to develop a method of direct calculation for the eigenfunctions of a large class of operators. Some of the results of this paper were discussed in a previous paper [5].

In Section 2, we show that a generalized path integral can be used to express the solution of a linear differential equation which has the interpretation of a function acted on by an infinitesimally generated transformation. In Section 3, we show that the path integral is an eigenfunction of the differential operator

* Supported in part by NSF Grant GP-43907.

Copyright (C) 1976 by Academic Press, Inc. 
generated by this transformation. Thus, to find eigenfunctions for a specified operator, we need only find a suitable generator. In Section 4, we show that, apart from questions of ordering the differential operators $q$ and $-i(\partial / \partial q)$, finding a suitable generator is equivalent to solving a related problem in classical mechanics. In Section 5, we solve the problem of ordering the operators $q$ and $-i(\partial / \partial q)$ for several special cases. In Section 6, we explicitly evaluate path integrals that have generators that are linear in either $q$ or $-i(\partial / \partial q)$. In Section 7, we develop a general technique for finding a generator which generates a specified operator. In Section 8, we illustrate our techniques by obtaining the eigenfunctions of several simple operators.

\section{The General Path Integral}

In this section we show that a generalized path integral can be used to express the solution of a differential equation which has the quantum-mechanical interpretation of a wavefunction acted on by an infinitesimally generated transformation. For simplicity and to illustrate the ideas involved, we work in one dimension; the general ideas are valid for an arbitrary number of degrees-of-freedom.

The differential equation we solve is

$$
G\left(q,-i \frac{\partial}{\partial q}, s\right) K\left(q, s ; q_{0}\right)=i \frac{\partial}{\partial s} K\left(q, s ; q_{0}\right)
$$

with the initial condition $K\left(q, s=0, q_{0}\right)=\delta\left(q-q_{0}\right)$. The differential operator $G$, which we call the generator, effects a one-parameter transformation of the function $K$, with $s$ the parameter of the transformation. A familiar example of Eq. (2.1) occurs when $G$ is the Hamiltonian operator and the parameter $s$ is the time. In this case, Eq. (2.1) is the Schrödinger equation and $K$ is the Green's function; the infinitesimally generated transformation is time translation. Equation (2.1) is the general statement for a transformation by an arbitrary operator.

We now write the solution $K(s)$ as a sequence of $N$ small transformations on $K(0)$. From Eq. (2.1) we see that

$$
K(s+\epsilon) \simeq\left[1-i \epsilon G\left(q,-i \frac{\partial}{\partial q}, s\right)\right] K(s)
$$

and setting $\epsilon=s / N$, we have by iteration

$$
\begin{aligned}
K(s) & =\lim _{N \rightarrow \infty}\left\{\prod_{k=N}^{1}\left[1-i \frac{s}{N} G\left(q,-i \frac{\partial}{\partial q}, \frac{(k-1) s}{N}\right)\right]\right\} K(0) \\
& \equiv U(s) K(0),
\end{aligned}
$$


where the product in Eq. (2.3) is written with index $k$ decreasing from left to right. We point out that the operator $U(s)$, which is defined in Eq. (2.3), cannot necessarily be defined as a quantum-mechanical operator which has a Hilbert space of functions as its domain and range; however, it can defined as an operator with a larger domain and range. We now demonstrate that $K(s)$ can be written as a path integral.

The conversion of Eq. (2.3) to a path integral formula is based on an identity for an operator function $F(q,-i(\partial / \partial q))$ acting on a general function $g(q)$. To derive the appropriate identity, we note that $g(q)$ can be written as

$$
g(q)=\lim _{\epsilon \rightarrow 0} \int d q^{\prime} \frac{d p}{2 \pi} e^{i p\left(q-q^{\prime}\right)-\epsilon p^{2}} g\left(q^{\prime}\right) .
$$

By doing the $p$-integration in Eq. (2.4) first, one can use thus identity for functions $g(q)$, which diverge at infinite $q^{2}$ as badly as $e^{\alpha q^{2}}$ for any positive $\alpha$. This ability to deal with badly divergent functions in the path integral generalization which we derive provides useful flexibility in applications. From Eq. (2.4), we derive the identity

$$
F\left(q,-i \frac{\partial}{\partial q}\right) g(q)=\lim _{\epsilon \rightarrow 0} \int d q^{\prime} \frac{d p}{2 \pi} F(q, p) e^{i p\left(q-q^{\prime}\right)-\epsilon p^{2}} g\left(q^{\prime}\right) .
$$

The formula given by Eq. (2.5) assumes that the derivatives $\partial / \partial q$ in the function $F$ do not act on the $q$-dependence of $F$. This is not the case in general. For many applications it is sufficient to assume that $F$ is a sum of symmetrized functions of $q$ and $-i(\partial / \partial q)$ of the form

$$
F\left(q,-i \frac{\partial}{\partial q}\right)=\frac{1}{2} \sum_{i}\left[f_{i}(q) g_{i}\left(-i \frac{\partial}{\partial q}\right)+g_{i}\left(-i \frac{\partial}{\partial q}\right) f_{i}(q)\right] .
$$

For an $F$ of the form (2.6) we find

$$
F\left(q,-i \frac{\partial}{\partial q}\right) g(q)=\frac{1}{2} \lim _{\epsilon \rightarrow 0} \int d q^{\prime} \frac{d p}{2 \pi}\left[F(q, p)+F\left(q^{\prime}, p\right)\right] e^{i p\left(q-q^{\prime}\right)-\epsilon p^{2}} g\left(q^{\prime}\right) .
$$

The path integral technique is a method for expressing Eq. (2.3) without the use of operators and by using ordinary functions of $q$ and $p$. The identity given by Eq. (2.5) is the device employed to replace operators by functions. Inserting Eq. (2.5) $N$ times into Eq. (2.3) we obtain

$$
\begin{aligned}
K\left(q, s ; q_{0}\right)= & \lim _{N \rightarrow \infty} \prod_{k=1}^{N} \int d q_{k-1} \frac{d p_{k}}{2 \pi} e^{i p_{k}\left(q_{k}-q_{k-1}\right)}\left[1-i \frac{s}{N} G\left(q_{k}, p_{k}, \frac{(k-1) s}{N}\right)\right] \\
& \times K\left(q_{0}, 0\right) .
\end{aligned}
$$


For $N$ large we can write

$$
1-i \frac{s}{N} G \simeq \exp \left(-i \frac{s}{N} G\right)
$$

and we can rewrite Eq. (2.8) in the form

$$
\begin{aligned}
K\left(q, s, q_{0}\right)= & \lim _{N \rightarrow \infty} \int \prod_{i=1}^{N-1} d q_{i} \prod_{j=1}^{N} \frac{d q_{j}}{2 \pi} \\
& \times \exp \left\{i \sum_{k=1}^{N}\left[p_{k}\left(q_{k}-q_{k-1}\right)-\frac{s}{N} G\left(q_{k}, p_{k},(k-1) \frac{s}{N}\right)\right]\right\} .
\end{aligned}
$$

Equation (2.10) is the path integral formula for $K\left(q, s ; q_{0}\right)$ in terms of the generating function $G$. If we generalize $G$ to operators of the form (2.6) we obtain

$$
\begin{aligned}
K\left(q, s ; q_{0}\right)= & \lim _{N \rightarrow \infty} \int \prod_{i=1}^{N-1} d q_{i} \prod_{j=1}^{N} \frac{d p_{i}}{2 \pi} \\
& \times \exp \left\{i \sum _ { k = 1 } ^ { N } \left[p_{k}\left(q_{k}-q_{k-1}\right)-\frac{s}{2 N} G\left(q_{k}, p_{k},(k-1) \frac{s}{N}\right)\right.\right. \\
& \left.\left.-\frac{s}{2 N} G\left(q_{k-1}, p_{k},(k-1) \frac{s}{N}\right)\right]\right\} .
\end{aligned}
$$

The standard shorthand notation for Eq. (2.11) is given by

$$
\begin{aligned}
K\left(q, s ; q_{0}\right)= & \int[d q]\left[\frac{d p}{2 \pi}\right] \\
& \times \exp \left\{i \int_{0}^{s} d s^{\prime}\left[p\left(s^{\prime}\right) \frac{d q\left(s^{\prime}\right)}{d s^{\prime}}-G\left(q\left(s^{\prime}\right), p\left(s^{\prime}\right), s^{\prime}\right)\right]\right\} .
\end{aligned}
$$

We close this section by emphasizing that in the derivation of Eq. (2.11), nowhere was it assumed that $G$ is a hermitian operator, nor does $K\left(q, s ; q_{0}\right)$ have to be square integrable-in fact, it may diverge as $e^{\alpha q^{2}}$ as $q^{2} \rightarrow \infty$.

\section{The Eigenfunction Equation}

In this section, we prove that $K\left(q, s ; q_{0}\right)$ is not only a solution to the differential equation (2.1), but $K$ is also the solution of another differential equation which shows that $K$ is an eigenfunction of a particular differential operator. 
Specifically, we prove that $K\left(q, s ; q_{0}\right)$ is a solution of the equation

$$
Q\left(q,-i \frac{\partial}{\partial q}, s\right) K\left(q, s ; q_{0}\right)=q_{0} K\left(q, s ; q_{0}\right),
$$

where $Q$ is the operator satisfying the differential equation

$$
\frac{d}{d s} Q\left(q,-i \frac{\partial}{\partial q}, s\right)=i\left[Q\left(q,-i \frac{\partial}{\partial q}, s\right), G\left(q,-i \frac{\partial}{\partial q}, s\right)\right],
$$

with the initial condition $Q(q,-i(\partial / \partial q), s=0)=q$.

The proof of Eq. (3.1) is accomplished by demonstrating that the solution of Eq. (3.2) can be written as

$$
Q(s)=U(s) q U^{-1}(s),
$$

where $U(s)$ is defined in Eq. (2.3). From Eqs. (2.3) and (2.1), we conclude that the operator $U(s)$ satisfies the same differential equation as $K$ :

$$
G(s) U(s)=i \frac{\partial}{\partial s} U(s) .
$$

By using Eq. (3.4) and the identity $d U^{-1} / d s=-U^{-1}(d U / d s) U^{-1}$, one can easily show that Eq. (3.3) is the solution of Eq. (3.2).

The importance of the eigenfunction equation (3.1) lies in the possibility of finding a generator $G$ which will lead (at some value of $s$, say, $s=1$ ) to a quantum operator $Q(s=1)$ of physical interest. For example, if $G$ can be selected such that $Q(s=1)$ is the Hamiltonian operator, then Eq. (2.12) becomes a path integral representation for energy eigenfunctions; furthermore, the fact that more than one $G$ can lead to the same $Q(s=1)$ implies a large amount of flexibility in practical calculations. We shall develop a general technique for finding operators $G$ which lead to a specified operator $Q(s=1)$. This general technique is developed on the basis of a close analogy with a related classical problem which we discuss in the next section.

\section{The Classical Problem}

In this section, we show that finding a generator $G$ such that the solution of Eq. (3.2) at $s=1$ is a specified function of $q$ and $-i(\partial / \partial q)$ is essentially equivalent to solving a similar problem in classical mechanics.

We define a classical "action" $S$ as

$$
S=\int_{0}^{s} d s^{\prime}\left[p\left(s^{\prime}\right) \frac{d q\left(s^{\prime}\right)}{d s^{\prime}}-\tilde{G}\left(q\left(s^{\prime}\right), p\left(s^{\prime}\right), s^{\prime}\right)\right]
$$


where $\tilde{G}$ is an arbitrary function of $q\left(s^{\prime}\right), p\left(s^{\prime}\right)$, and $s^{\prime}$. The requirement that $S$ be stationary with respect to small variations of $q\left(s^{\prime}\right)$ and $p\left(s^{\prime}\right)$, subject to the constraints $\delta q(s)=\delta q(0)=0$, leads to the following "Hamilton's" equations for $q\left(s^{\prime}\right)$ and $p\left(s^{\prime}\right)$

$$
\frac{d q\left(s^{\prime}\right)}{d s^{\prime}}=\frac{\partial \tilde{G}}{\partial p}\left(q\left(s^{\prime}\right), p\left(s^{\prime}\right), s^{\prime}\right)
$$

and

$$
\frac{d p\left(s^{\prime}\right)}{d s^{\prime}} \equiv-\frac{\partial \tilde{G}}{\partial q}\left(q\left(s^{\prime}\right), p\left(s^{\prime}\right), s^{\prime}\right) .
$$

These equations can be written in Poisson bracket notation as follows:

$$
d q\left(s^{\prime}\right) / d s^{\prime}=\left\{q\left(s^{\prime}\right), \tilde{G}\left(q\left(s^{\prime}\right), p\left(s^{\prime}\right), s^{\prime}\right)\right\}
$$

and

$$
d p\left(s^{\prime}\right) / d s^{\prime}=\left\{p\left(s^{\prime}\right), \widetilde{G}\left(q\left(s^{\prime}\right), p\left(s^{\prime}\right), s^{\prime}\right)\right\} .
$$

If $G$ is not explicitly $s$-dependent, the classical analog of the quantum equation (3.2) is given by Eq. (4.3a) with $\widetilde{G}=-G$. (The minus sign is a result of the fact that Eq. (3.2) differs by a minus sign from the usual "Heisenberg" dynamical operator equation.) If $G$ is $s$-dependent, care must be taken in comparing Eq. (4.3a) with Eq. (3.2). The important point is that the $q$ and $p$ operators appearing as arguments of $G$ in Eq. (3.2) are evaluated at $s=0$, whereas the classical quantities appearing as arguments of $G$ in Eq. (4.3a) are evaluated at $s^{\prime}$.

As shown in the appendix, if the solutions of Eqs. (4.2) are such that $q(1)=f[q(0), p(0)]$, then the solution at $s=1$ of Eq. (3.2) with $G(q,-i(\partial / \partial q), s)=$ $-\tilde{G}(q,-i(\partial / \partial q), 1-s)$ is given by

$$
Q\left(q,-i \frac{\partial}{\partial q}, s=1\right)=f\left[q,-i \frac{\partial}{\partial q}\right]
$$

However, if $\bar{q}\left(s^{\prime}\right)$ and $\bar{p}\left(s^{\prime}\right)$ are solutions of Eq. (4.2), then $q\left(s^{\prime}\right)=\bar{q}\left(1-s^{\prime}\right)$ and $p\left(s^{\prime}\right)=\bar{p}\left(1-s^{\prime}\right)$ are solutions of the equations

$$
\frac{d q\left(s^{\prime}\right)}{d s^{\prime}}=\frac{\partial G}{\partial p}\left(q\left(s^{\prime}\right), p\left(s^{\prime}\right), s^{\prime}\right),
$$

and

$$
\frac{d p\left(s^{\prime}\right)}{d s^{\prime}}=-\frac{\partial G}{\partial q}\left(q\left(s^{\prime}\right), p\left(s^{\prime}\right), s^{\prime}\right),
$$

where $G(q, p, s)=-\tilde{G}(q, p, 1-s)$. Thus, the classical generator $G(q, p, s)$ effects a transformation that is the inverse of the transformation effected by the quantum 
generator $G(q,-i(\partial / \partial q), s)$ in the sense that, if $Q(q,-i(\partial / \partial q), s=1)$ is the solution of Eq. (3.2), then the solutions of Eq. (4.4) are such that $q(0)=Q(q(1), p(1), s=1$ ).

Therefore, to find a quantum generator $G(q,-i(\partial / \partial q), s)$ which gives $Q(q,-i(\partial / \partial q), s=1)$ as a specified function of $q$ and $-i(\partial / \partial q)$, one finds a classical generator $G(q, p, s)$ which gives $q(0)$ as the same function of $q(1)$ and $p(1)$; such a classical generator can always be found, as explained in Section 7. The classical generator is inserted directly into the path integral given by Eq. (2.12). There remains, of course, the question of how to order position and momentum operators in going from the classical to the quantum problem; we discuss this question in the next section.

\section{THE ORDERING OF THE OPERATORS}

In this section, we show that the problem of ordering the position and momentum operators in going from the classical to the quantum generator $G$ is particularly simple in two cases: (1) $G$ depends linearly on either $q$ or $p$; (2) $G$ depends quadratically on $q$ and $p$.

The solution of Eq. (3.2) can be written as an infinite sum of iterated commutators. This expression is given by

$$
\begin{aligned}
Q\left(q,-i \frac{\partial}{\partial q}, s\right)= & q+i \int_{0}^{s} d s_{1}\left[q, G\left(q,-i \frac{\partial}{\partial q}, s_{1}\right)\right] \\
& +i^{2} \int_{0}^{s} d s_{1} \int_{0}^{s_{1}} d s_{2}\left[\left[q, G\left(q,-i \frac{\partial}{\partial q}, s_{2}\right)\right], G\left(q,-i \frac{\partial}{\partial q}, s_{1}\right)\right] \\
& +\cdots .
\end{aligned}
$$

We first consider the case in which $G$ is linear in $q$. We assume $G$ to be symmetized, i.e.,

$$
G\left(q,-i \frac{\partial}{\partial q}, s\right)=\frac{1}{2}\left[q g\left(-i \frac{\partial}{\partial q}, s\right)+g\left(-i \frac{\partial}{\partial q}, s\right) q\right] .
$$

In this case, each term in Eq. (5.1) is linear in $q$ and symmetrized with respect to $-i(\partial / \partial q)$. The operator $Q(q,-i(\partial / \partial q), s=1)$ can thus be deduced from the solution of the classical equations (4.4), which will be linear in $q(1)$. One simply inserts operators for the classical quantities and symmetrizes.

In the case that $G$ is a linear function of $p$, each term in Eq. (5.1) will be a function of $q$ only. In the case where $G$ is a quadratic function of both $q$ and $-i(\partial / \partial q)$, each term in Eq. (5.1) is linear in $q$ and $-i(\partial / \partial q)$. In both these cases, no question of ordering can arise and the operator $Q(q,-i(\partial / \partial q), s=1)$ is obtained by straightforward solution of the classical equations (4.4). 
More complicated $G$ 's must be studied separately, but a fairly simple relation between the quantum and classical operators may presumably be anticipated whenever $G$ is a polynomial. Fortunately, even the restriction of $G$ to linear and quadratic functions of $q$ and $-i(\partial / \partial q)$ permits the solution of some problems of interest.

\section{Evaluation of Path Integrals with Linear Generators}

In the case where $G$ is linear in either $p$ or $q$, the path integral can be reduced to a particularly simple form.

We first assume that $G$ is of the form

$$
G\left(q,-i \frac{\partial}{\partial q}, s\right)=\frac{1}{2} q f\left(-i \frac{\partial}{\partial q}, s\right)+\frac{1}{2} f\left(-i \frac{\partial}{\partial q}, s\right) q .
$$

The corresponding classical generator $G(q, p, s)=q f(p, s)$ yields the following equations for $q(s)$ and $p(s)$

$$
\frac{d q}{d s}=q(s) \frac{\partial f}{\partial p}(p(s), s)
$$

and

$$
\frac{d p}{d s}=-f(p(s), s)
$$

We note that the solution of Eq. (6.3) is completely determined by the value of $p(s=1)$. We also note that the solution of Eq. (6.2) is given by

$$
q(s)=q(1) \exp \left[\int_{1}^{s} d s^{\prime} \frac{\partial f}{\partial p}\left(p\left(s^{\prime}\right), s^{\prime}\right)\right] \equiv q(1) h(s ; p(s=1)) .
$$

At $s=1$, the path integral for the generator given by Eq. (6.1) becomes

$$
\begin{aligned}
K\left(q, s=1 ; q_{0}\right)= & \lim _{N \rightarrow \infty} \int \prod_{i=1}^{N-1} d q_{i} \prod_{j=1}^{N} \frac{d p_{j}}{2 \pi} \\
& \left.\times \exp \left\{i \sum_{k=1}^{N} p_{k}\left(q_{k}-q_{k-1}\right)-\frac{q_{k}+q_{k-1}}{2 N} f\left(p_{k}, \frac{k-1}{N}\right)\right]\right\}
\end{aligned}
$$


Each of the $q_{i}$ integrations in Eq. (6.2) yields a delta-function

$$
\begin{aligned}
K(q, s= & \left.1 ; q_{0}\right) \\
= & \lim _{N \rightarrow \infty} \frac{1}{2 \pi} \int \prod_{\jmath=1}^{N} d p_{j} e^{i \alpha\left(p_{N}, q, p_{1}, q_{0}\right)} \\
& \times \prod_{k=1}^{N-1} \delta\left[p_{k}-p_{k+1}-\frac{1}{2 N} f\left(p_{k+1}, \frac{k}{N}\right)-\frac{1}{2 N} f\left(p_{k}, \frac{k-1}{N}\right)\right],
\end{aligned}
$$

where

$$
\alpha\left(p_{N}, q, p_{1}, q_{0}\right)=p_{N} q-p_{1} q_{0}-\frac{1}{2 N}\left[q f\left(p_{N}, 1-\frac{1}{N}\right)+q_{0} f\left(p_{1}, 0\right)\right] .
$$

In the limit $N \rightarrow \infty$, the vanishing of the arguments of the delta-functions in Eq. (6.6) can be expressed as

$$
-d p-\frac{1}{2} d s[f(p(s), s)+f(p(s+d s), s+d s)]=0 .
$$

To first order in $d s$, Eq. (6.7) is identical to Eq. (6.3); this fact simplifies the evaluation of the $p$-integrations. Integrating over $p_{1} p_{2} \cdots p_{n-1}$ in Eq. (6.6), we obtain (including the Jacobian for each integration)

$$
K\left(q, s=1 ; q_{0}\right)=\lim _{N \rightarrow \infty} \frac{1}{2 \pi} \int d p_{N} e^{i \alpha} \prod_{k=1}^{N-1}\left|1-\frac{1}{2 N} \frac{\partial f}{\partial p}\left(p_{k}, \frac{k-1}{N}\right)\right|^{-1},
$$

where $p_{k}$ is the value of $p(s=k / N)$ on the classical trajectory determined by $p(s=1)=p_{N}$. As $N \rightarrow \infty$ the terms proportional to $1 / N$ in $\alpha$ vanish and the Jacobian in Eq. (6.8) can be written in exponential form. We obtain the final result

$$
\begin{aligned}
& K\left(q, s=1 ; q_{0}\right) \\
& =\frac{1}{2 \pi} \int d p_{N} \exp \left(i q p_{N}-i q_{0} p(s=0)+\frac{1}{2} \int_{0}^{1} d s \operatorname{Re} \frac{\partial f}{\partial p}(p(s), s)\right) \\
& =\frac{1}{2 \pi} \int d p_{N}\left|h\left(0, p_{N}\right)\right|^{-1 / 2} \exp \left(i q p_{N}-i q_{0} p(s=0)\right),
\end{aligned}
$$

where $h$ is defined in Eq. (6.4). The region of integration in Eq. (6.9) is the set of values of $P_{N}$ for which $p(s=0)$ is real. We note that $K\left(q, s=1, q_{0}\right)$ depends only upon the classical equations relating $q(0)$ and $p(0)$ to $q(1)$ and $p(1)$.

We now assume that $G$ is of the form

$$
G\left(q,-i \frac{\partial}{\partial q}, s\right)=\frac{1}{2}\left[-i \frac{\partial}{\partial q} \bar{f}(q, s)-i \bar{f}(q, s) \frac{\partial}{\partial q}\right] .
$$


The corresponding classical generator $G(q, p, s)=p \bar{f}(q, s)$ yields the following equations for $q(s)$ and $p(s)$ :

$$
d q / d s=\bar{f}(q(s), s)
$$

and

$$
\frac{d p}{d s}=-p(s) \frac{\partial \bar{f}}{\partial q}(q(s), s) .
$$

The solution of Eq. (6.11) is completely determined by the value of $q(s=1)$ and the solution of Eq. (6.12) is given by

$$
p(s)=p(1) \exp \left[-\int_{1}^{s} \bar{f}\left(q\left(s^{\prime}\right), s^{\prime}\right) d s^{\prime}\right] \equiv p(1) \bar{h}(s ; q(1)) .
$$

The evaluation of the path integral for the generator given by Eq. (6.10) is similar to the evaluation of the path integral for the case in which $G$ is linear in $q$. The final result is given by

$$
K\left(q, s=1 ; q_{0}\right)=\exp \left(-\frac{1}{2} \int_{0}^{1} d s \operatorname{Re} \frac{\partial \bar{f}}{\partial q}(q(s), s)\right) \delta\left(q_{0}-q(s=0)\right),
$$

where $q(s=0)$ is evaluated on the classical trajectory determined by $q(s=1)=q$. We note that $K\left(q, s=1 ; q_{0}\right)$ depends only upon the classical equations relating $q(0)$ and $p(0)$ to $q(1)$ and $p(1)$.

\section{A General Technique for Finding the Generator}

In order to make practical use of Eq. (3.1) as an eigenfunction equation, a technique is needed for finding a generator $G$ which will generate a desired operator $Q(q,-i(\partial / \partial q), s)$. Because of the close analogy between the quantum and classical problems, it is possible to use the classical theory as an aid to discovering the desired G. Instead of working directly with the equations of motion (4.4), it is useful to appeal to the general theory of canonical transformations. Thus, we regard $q(s)$ and $p(s)$ as related to $q(1)$ and $p(1)$ by means of an infinitesimally generated canonical transformation. The validity of the view stems from Eqs. (4.4), which are just the equations for an infinitesimally generated canonical transformation.

We define a so-called generating function $F_{1}(q(s), q(1), s)$ for the canonical transformation in terms of the solutions of Eqs. (4.4) by

$$
F_{1}(q(s), q(1), s) \equiv \int_{s}^{1} d s^{\prime}\left[p\left(s^{\prime}\right) \frac{d q\left(s^{\prime}\right)}{d s^{\prime}}-G\left(q\left(s^{\prime}\right), p\left(s^{\prime}\right), s^{\prime}\right)\right] .
$$


The generating function $F_{1}$ depends only upon endpoint values of $q$, i.e., $q(s)$ and $q(1)$ because the classical path $\left(q\left(s^{\prime}\right)\right.$ and $\left.p\left(s^{\prime}\right)\right)$ is completely determined by $q(s)$ and $q(1)$. The canonical transformation $\{q(1), p(1)\} \rightarrow\{q(s), p(s)\}$ is obtained by differentiating $F_{1}$ with respect to its arguments as follows:

$$
p(s)=-\partial F_{1} / \partial q(s), \quad p(1)=\partial F_{1} / \partial q(1) .
$$

The generator $G$ can be recovered from $F_{1}$ through the equation

$$
\partial F_{1} / \partial s=G
$$

For applications it is somewhat more convenient to define another generating function $F_{2}(q(1), p(s), s)$ written as a function of $q(1), p(s)$ and $s$ and related to $F_{1}$ by

$$
F_{2}(q(1), p(s), s)=F_{1}(q(s), q(1), s)+p(s) q(s) .
$$

From Eq. (7.4), we can deduce that

$$
p(1)=\partial F_{2} / \partial q(1), \quad q(s)=\partial F_{2} / \partial p(s),
$$

and

$$
\partial F_{2} / \partial s=G \text {. }
$$

The generating function $F_{2}$ has the useful property that

$$
F_{2}{ }^{I}=q(1) p(s)
$$

gives rise to the identity transformation. In general, it is simple to determine the generator $G$ by means of Eq. (7.6) from an appropriate $F_{2}$. It should be noted that Eqs. (7.5) can be used to determine $q(s)$ and $p(s)$ as functions of $q(1), p(1)$, and $s$. Then $q(s)$ and $p(s)$ will satisfy Eq. (6.4) with $G$ given by Eq. (7.3).

To determine an appropriate generator $G$, we first find a function $\bar{F}_{1}(q(0), q(1))$ such that if we invert the equation

$$
p(1)=\partial \bar{F}_{1} / \partial q(1)
$$

to express $q(0)$ in terms of $q(1)$ and $p(1)$, then $q(0)$ is the classical quantity corresponding to the desired quantum mechanical operator $Q(q,-i(\partial / \partial q), s=1)$. We then invert the equation

$$
p(0)=-\partial \bar{F}_{1} / \partial q(0)
$$

to express $q(0)$ in terms of $q(1)$ and $p(0)$

$$
q(0)=h(q(1), p(0))
$$


We then define a related function $\bar{F}_{2}(q(1), p(0))$ by

$$
\bar{F}_{2}(q(1), p(0))=\bar{F}_{1}[h(q(1), p(0)), p(0)]+p(0) h(q(1), p(0)) .
$$

Finally, we form the desired generating function $F_{2}(q(1), p(s), s)$ as follows:

$$
F_{2}(q(1), p(s), s)=s q(1) p(s)+(1-s) \bar{F}_{2}(q(1), p(s)) .
$$

The particular construction of Eq. (7.11) guarantees that the transformation will be the identity for $s=1$ and the desired transformation for $s=0$. The generator $G$ is obtained from Eq. (7.6). The resulting $G$ is clearly not unique.

\section{EXAMPLES}

In this section, we illustrate our techniques by using them to find eigenfunctions of several simple operators.

\section{A. The Momentum Operator, $-i(\partial / \partial q)$}

For this case, we can instantaneously determine a suitable classical generator given by

$$
G(q, p, s)=-(\pi / 4)\left(q^{2}+p^{2}\right) .
$$

When this generator is inserted into the right-hand side of Eq. (2.11), the resulting path integral is gaussian and can be evaluated by well-known techniques. The result is given by

$$
K\left(q, s=1 ; q_{0}\right)=(i /(2 \pi))^{1 / 2} e^{i q_{0} q} .
$$

The fact that Eq. (8.2) is correctly (continuum) normalized is a consequence of the reality of the generator.

B. The Operator $Q=f(q)$

A suitable classical $\bar{F}_{2}$ for this case is given by

$$
\bar{F}_{2}(q(1), p(0))=p(0) f(q(1)) .
$$

The equations relating $p(0)$ and $q(0)$ to $p(1)$ and $q(1)$ are given by

and

$$
p(1)=\partial \bar{F}_{2} / \partial q(1)=p(0) f^{\prime}(q(1)),
$$

$$
q(0)=\partial \bar{F}_{2} / \partial p(0)=f(q(1)) .
$$

Since $\bar{F}_{2}$ is a linear function of $p(0)$, the generator $G(q, p, s)$ which can be obtained from $\bar{F}_{2}$ by the techniques of Section 7 is guaranteed to be linear in $p$. The path 
integral can therefore be evaluated using the techniques of Section 6 . The result is given by

$$
K\left(q, s=1 ; q_{0}\right)=\left|f^{\prime}(q)\right|^{1 / 2} \delta\left[q_{0}-f(q)\right] .
$$

We note that we did not need to explicitly find $G(q, p, s)$ in order to arrive at Eq. (8.6).

More generally, we may take $F_{2}$ to be

$$
\bar{F}_{2}(q(1), p(0))=p(0) f(q(1))+g(q(1))
$$

where $g(q)$ is an arbitrary function. The only effect of the extra term in Eq. (8.7) upon the resulting path integral is to multiply the right-hand side of Eq. (8.6) by a $q$-dependent factor:

$$
K\left(q, s=1 ; q_{0}\right)=e^{-i g(q)}\left|f^{\prime}(q)\right|^{1 / 2} \delta\left[q_{0}-f(q)\right] .
$$

We have thus obtained the most general eigenfunction of the operator $f(q)$. We believe that this example illustrates a general property of our technique, i.e., that the arbitrariness in the choice of the generator (or the generating function) can be exploited to obtain the most general solution of Eq. (3.1).

\section{The Free Particle Hamiltonian}

We may combine the results of the previous two subsections to obtain the eigenfunctions of the free particle Hamiltonian

$$
H=-\frac{1}{2 m} \frac{\partial^{2}}{\partial q^{2}} .
$$

Specifically, we choose the classical generator to be

$$
G(q, p, s)=\theta\left(s-\frac{1}{2}\right) G_{1}(q, p, s)+\theta\left(\frac{1}{2}-s\right) G_{2}(q, p, s),
$$

where $\theta(x)$ is the Heaviside step-function, $G_{1}(q, p, s)$ is a classical generator which effects a transformation for which $q\left(\frac{1}{2}\right)=p(1)$, and $G_{2}(q, p, s)$ is a classical generator which effects a transformation for which $q(0)=(1 / 2 m)\left[q\left(\frac{1}{2}\right)\right]^{2}$. The resultant path integral is the convolution of two intermediate path integrals each of which can be explicitly evaluated by a simple variation of the results of the previous subsections. The result is given by

$$
\begin{aligned}
K\left(q, s=1 ; q_{0}\right)= & \int d q^{\prime}\left[\left(\frac{i}{2 \pi}\right)^{\mathbf{1 / 2}} e^{i q q^{\prime}}\right]\left[e^{-i g\left(q^{\prime}\right)} \frac{\left|q^{\prime}\right|}{m} \delta\left(q_{0}-\frac{q^{\prime 2}}{2 m}\right)\right] \\
= & \left(\frac{i}{2 \pi}\right)^{\mathbf{1 / 2}}\left[\exp \left(i\left(2 m q_{0}\right)^{1 / 2} q-i g\left[\left(2 m q_{0}\right)^{1 / 2}\right]\right)\right. \\
& +\exp \left(-i\left(2 m q_{0}\right)^{\mathbf{1} / 2} q-i g\left[-\left(2 m q_{0}\right)^{\mathbf{1} / 2}\right]\right),
\end{aligned}
$$

where $g(x)$ is an arbitrary function. 
D. The Operator $Q=(1 / 2) q f(-i(\partial / \partial q))+(1 / 2) f(-i(\partial / \partial q)) q$

A suitable classical $\bar{F}_{2}$ for this case is given by

$$
\bar{F}_{2}(q(1), p(0))=q(1) y[p(0)],
$$

where $y(x)$ satisfies the equation

$$
x=\int^{y(x)} \frac{d w}{f(w)} .
$$

The following differential equation can be derived from Eq. (8.13):

$$
d y / d x=f[y(x)]
$$

The equations relating $p(0)$ and $q(0)$ to $p(1)$ and $q(1)$ are given by

$$
p(1)=\partial \bar{F}_{2} / \partial q(1)=y[p(0)],
$$

and

$$
q(0)=\partial \bar{F}_{2} / \partial p(0)=\left.q(1)(d y / d x)\right|_{x=p(0)} .
$$

We can use Eqs. (8.14) and (8.15) to rewrite Eq. (8.16) as

$$
q(0)=q(1) f(p(1)) \text {. }
$$

The generator which is obtained from $\bar{F}_{2}$ by the techniques of Section 7 is linear in $q$ :

$$
G(q, p, s)=q[p-y(p)]\left[s+(1-s) y^{\prime}(p)\right]^{-1}
$$

Therefore the resultant path integral can be evaluated by using the techniques of Section 6; the result is given by

$$
K\left(q, s=1 ; q_{0}\right)=\frac{1}{2 \pi} \int d p|f(p)|^{-1 / 2} e^{i q p-i q_{0} z(p)},
$$

where

$$
z(p)=\int^{p} \frac{d w}{f(w)}
$$

As an example, we choose $f(w)=w$. For this example, $z(p)=\ln p$ and the path integral is given by

$$
K\left(q, s=1 ; q_{0}\right)=\frac{1}{2 \pi} \int_{0}^{\infty} d p p^{-i q_{0}-1 / 2} e^{i q p} .
$$


E. The Harmonic Oscillator Hamiltonian

A convient choice for the classical generator is given by

$$
G(q, p, s)=\theta\left(s-\frac{1}{2}\right) \frac{\pi}{4}\left(\frac{q^{2}}{\lambda}+\lambda p^{2}\right)+\theta\left(\frac{1}{2}-s\right) \bar{G}(q, p, s),
$$

where $\lambda$ is an arbitrary parameter and $\bar{G}(q, p, s)$ is a classical generator which effects a transformation for which $q(0)=q\left(\frac{1}{2}\right) p\left(\frac{1}{2}\right)$. The classical generator $(\pi / 4)\left(\left(q^{2} / \lambda\right)+\lambda p^{2}\right)$ effects a transformation for which

$$
q\left(\frac{1}{2}\right)=\frac{1}{\sqrt{2}}[q(1)-\lambda p(1)] \quad \text { and } \quad p\left(\frac{1}{2}\right)=\frac{1}{\sqrt{2}}\left[\frac{1}{\lambda} q(1)+p(1)\right] .
$$

Thus, the generator given by Eq. (8.22) generates a canonical transformation for which

$$
q(0)=\frac{1}{2 \lambda} q^{2}(1)-\frac{1}{2} \lambda p^{2}(1) .
$$

Furthermore, by using the results of Section 5, we can easily check that when the $G$ given by Eq. (8.22) is inserted into (2.11), the resultant path integral is an eigenfunction of the operator

$$
Q\left(q,-i \frac{\partial}{\partial q}, s=1\right)=\frac{1}{2}\left(\lambda \frac{\partial^{2}}{\partial q^{2}}+\frac{1}{\lambda} q^{2}\right) .
$$

For $\lambda= \pm i$, the right-hand side of Eq. (8.24) is a multiple of the Hamiltonian operator for a harmonic oscillator with unit mass and spring constant.

The resultant path integral $K\left(q, s=1, q_{0}\right)$ is the convolution of two simple intermediate path integrals of which one is gaussian and the other is given by Eq. (8.21). The overall result is given by

$$
\begin{aligned}
K\left(q, s=1 ; q_{0}\right)= & \int_{-\infty}^{+\infty} d q^{\prime}\left\{\left((2 \pi i)^{1 / 2} \lambda\right)^{-1 / 2} \exp \left[\frac{i}{\lambda}\left(\frac{q^{2}+q^{\prime 2}}{2}-\sqrt{2} q q^{\prime}\right)\right]\right\} \\
& \times\left\{\frac{1}{2 \pi} \int_{0}^{\infty} d p p^{-i q_{0}-1 / 2} e^{i q^{\prime} p}\right\} .
\end{aligned}
$$

We may carry out the intermediate $q^{\prime}$ integration to obtain

$$
K\left(q, s=1 ; q_{0}\right)=\frac{2^{-3 / 4}}{\pi} \int_{0}^{\infty} d p p^{-1 / 2-i q_{0}} \exp \left\{-\frac{i}{2 \lambda} q^{2}+i \sqrt{2} q p-\frac{i}{2} \lambda p^{2}\right\} .
$$

In deriving Eq. (8.26) we have assumed that $\lambda$ is in the lower half-plane and $q_{0}$ 
is real. However, we may analytically continue $K$ into the entire $q_{0}$ and $\lambda$ planes by writing it in the form

$$
\begin{aligned}
K\left(q, s=1 ; q_{0}\right)= & \frac{2^{-7 / 4}}{\pi} \frac{e^{-\pi q_{0}}}{\sin \pi\left(\frac{1}{2}+i q_{0}\right)} \int_{c} d p p^{-1 / 2-i q_{0}} \\
& \times \exp \left\{-\frac{i}{2 \lambda} q^{2}+i \sqrt{2} q p-\frac{i}{2} \lambda p^{2}\right\},
\end{aligned}
$$

where the contour circles the singularity at zero and goes to infinity within the wedge

$$
-\frac{\pi}{2}-\arg \frac{\lambda}{2}<\arg p<-\arg -\frac{\lambda}{2} .
$$

In the complex $q_{0}$-plane, $K\left(q, s=1, q_{0}\right)$ has poles at the points $q_{0}=-i n-\frac{1}{2}$ $(n=0,1,2, \ldots)$. At these points the contour integral appearing in Eq. (8.27) can be expressed in terms of Hermite polynomials. The residues at the poles in $K\left(q, s=1, q_{0}\right)$ are given by

$$
\begin{aligned}
& \lim _{q_{0} \rightarrow-i(n+1 / 2)}\left[q_{0}+i\left(n+\frac{1}{2}\right)\right] K\left(q, s=1 ; q_{0}\right) \\
& \quad=-\frac{1}{n !}\left(\frac{i}{2} \lambda\right)^{n / 2} \frac{2^{-3 / 4}}{\pi} e^{-(i / \lambda 2) q^{2}} H_{n}\left[\frac{q}{(i \lambda)^{1 / 2}}\right] .
\end{aligned}
$$

For $\lambda=i$, the differential operator

$$
\frac{1}{2}\left(\lambda \frac{\partial^{2}}{\partial q^{2}}+\frac{1}{\lambda} q^{2}\right)
$$

is equal to $-i H$, where $H$ is the harmonic oscillator Hamiltonian. Identifying $q_{0}$ with $-i E$, we recognize that the right-hand side of Eq. (8.28) gives the correct (unnormalized) harmonic oscillator eigenstates in the case $\lambda=i$. However, for values of $q_{0}$ away from the pole position in the case $\lambda=+i$, and for all values of $q_{0}$ in the case $\lambda=-i, K\left(q, s=1, q_{0}\right)$ cannot be interpreted as a harmonic oscillator energy eigenstate because it diverges too rapidly as $q$ approaches infinity.

\section{APPENDIX}

In this appendix, we prove the result quoted in Section 4 , that $-G(q,-i(\partial / \partial q)$, $1-s$ ) is the appropriate quantum operator for Eq. (3.2). To establish this result we consider the following equations for operators $F(s)$ and $\bar{F}(s)$ :

$$
\frac{d F(s)}{d s}=i\left[F(s), G\left(q,-i \frac{\partial}{\partial q}, s\right)\right],
$$


where from Section 3 we know that the solution to (A.1) can be written

with

$$
F(s)=U(s) F(0) U^{-1}(s), \quad U(0)=I,
$$

$$
G(s) U(s)=i(\partial / \partial s) U(s)
$$

$\bar{F}(s)$ satisfies the related equation

where

$$
d \bar{F}(s) / d s=i[\bar{F}(s), G(Q(s), P(s), 1-s)]
$$

$$
\begin{aligned}
\bar{F}(s) & =V^{-1}(s) \bar{F}(0) V(s), \quad V(0)=I \\
G(Q(s), P(s), 1-s) & =V^{-1}(s) G\left(q,-i \frac{\partial}{\partial q}, 1-s\right) V(s),
\end{aligned}
$$

and

$$
G\left(q,-i \frac{\partial}{\partial q}, 1-s\right) V(s)=-i \frac{\partial}{\partial s} V(s) .
$$

From (A.5) and (A.3) we can conclude that

$$
V(s)=U(1-s) U^{-1}(1)
$$

is a solution to (A.6).

Now we see that for the finite transformation $(s=1)$ we have the result from (A.7) that

$$
\bar{F}(s=1)=F(s=1) \quad \text { if } \quad \bar{F}(s=0)=F(s=0) .
$$

Thus, if we are interested in the finite transformation to $s=1$ given by the solution to (4.4) we can equally well see (A.4) setting $\bar{F}(s)=Q(s)$. However, (A.4) has the corresponding classical equation:

$$
\frac{d q(s)}{d s}=-\frac{\partial G}{\partial p}(q(s), p(s), 1-s),
$$

which corresponds to the result we wished to prove.

\section{REFERENCES}

1. R. P. Feynman And A. R. HibBs, "Quantum Mechanics and Path Integrals," MacGraw-Hill, New York, 1965.

2. E. S. Abers ANd B. W. Lee, Phys. Repts. 9C, No. 1 (1973).

3. C. Garrod, Rev. Mod. Phys. 38 (1966), 483.

4. M. C. Gutzwiller, J. Math. Phys. 10 (1969), 1004; J. Math. Phys. 8 (1967), 1979.

5. W. B. Campbell, P. Finkler, C. E. Jones, AND M. N. Misheloff, Path integral formulation for eigenfunctions, Nuovo Cimento, to appear. 